\title{
MONITORING OF SUSPENDED SUBSTANCE CONCENTRATION IN THREE GORGES DAM BASED ON THE SEMI- ANALYTICAL MODEL
}

\author{
GAI Li-ya ${ }^{\text {a, b }}$, LIU Zheng-jun ${ }^{\mathrm{a}}$,ZHANG Ji-xian ${ }^{\mathrm{c}, \mathrm{a}}$,Sun Yin-hang ${ }^{\mathrm{b}}$ \\ ${ }^{\text {a }}$ Key Laboratory of Mapping from Space of State Bureau of Surveying and Mapping, Chinese Academy of Surveying \\ and Mapping, Beijing 100039,China-zjliu @casm.ac.cn \\ ${ }^{\mathrm{b}}$ Center for Hydrogeology and Environmental \\ Geology,CGS,Baoding,071051,China-(gailiya1982,sunyinhang)@163.com \\ c.Chinese Academy of Surveying and Mapping, Beijing 100039,China- zhangjx@casm.ac.cn
}

KEY WORDS: Chlorophyll a; Semi-analytical model; Retrieval; Three Gorges Dam

\begin{abstract}
:
Chlorophyll a (chla) concentration is an important index of water quality evaluation. It is significant to build a quantitative remote sensing retrieval algorithm to inverse the chla concentration. With focus on the problems that empirical method is lack of the support of the physical theory and not robust in quantitative remote sensing monitor of SS concentration in inland water, the feasibility of spectrum and inherent optical properties analysis with clear physical meaning is discussed. Based on the experiment taken on 17th, November 2007, the characteristic spectrum, absorption coefficient and chla concentration are taken on 30 samples distributed along the reservoir where is far away from the dam above 3 kilometers. The absorption characteristic of the chlorophyll a and the contribution of the main constituents of the water (chlorophyll a, suspended substance, DOC) to the total absorption are analyzed. Based on the achievements we have acquired, two semi-analytical models using Gordon model is built to inverse the chla concentration. With the chla concentration on samples, the precision of the inversion models are tested. The results show that the no more than $30 \%$ of the inversion data whose estimation precision were over $70 \%$.Further, the inversion method is applied on the multi-temporal image CHRIS to check the prison of the model, and the mean retrieval error is $50.17 \%$.In despite of the low retrieval precision, the chlorophyll a concentration retrieval model is valuable in the remote sensing monitoring of the inland water quality.

Many empirical/semi-analytical/analysis algorithms of inversing chlorophyll a concentration have been developed since the CZCS (costal zone color scanner) operates. The empirical statistic algorithms based on the "band ratio /combination" , the precision of which are higher in case I water, become the main algorithms on business(Ren Jing-ping,2002). For case II water, the inversion precision can increased by choosing the appropriate bands and increasing the spectrum bands. These algorithms not only apply simply, but also calculate speedy. But there is a drawback that the applicability of them is worse. Semi-analytical algorithms which are based on the water color principle, integrate the empirical or subjective hypothesis values in the bio-optical model, using the bio-optical model to interpret or model the remote sensing data, then inverse the water component concentration from the remote sensing data by AOP band ratio and mathematics methods (Liu Chan-de, 2005). Semi-analytical algorithms are suitable for inland water color remote sensing monitoring due to its less dependency of the ground in-situ data. Especially with the development of the highspectral sensor technology and the optical instrument in ocean and lake, Semi-analytical algorithms play an important role in inland water color remote sensing monitoring. In this paper, we established the Semi-analytical algorithm of the Three Gorges Dam water using the Gordon model, then inversed the water components concentration with the CHRIS data.
\end{abstract}

\section{WATER COLOR PRINCIPLE AND ALGORITHM DEVELOP}

1.1 The Relationship between the Water Color and the Absorption \& Scatter of Water Components

The optical characteristic of water is related to its components. Chlorophyll, dissolved organic carbon (DOC) and suspended substance (SS), which are the so called "water color three components" without considering the water bubble, turbulent current effect and other factors, are the three factors to influence the case II water optical characteristic, and the last factor is the pure water. The inherent optical properties (absorption coefficient, scatter coefficient, attenuation coefficient) are the combination of the four components (Li Tong-ji, 2006). Therefore, the water total absorption coefficient $a$ and total back-scatter coefficient $b_{b}$ are the total of the different absorption bodies and scatter bodies' coefficients:

$$
\begin{aligned}
& a=a_{w}+a_{p h}+a_{d}+a_{g} \\
& b_{b}=b_{b_{w}}+b_{b_{p h}}+b_{b_{d}}+b_{b_{g}}
\end{aligned}
$$

$a_{w}, a_{p h}, a_{d}, a_{g}$ is the absorption coefficient of the pure water, chlorophyll, SS and DOC respectively; $b_{b w}{ } b_{b_{p h}}$,

$b_{b_{d}}, b_{b_{g}}$ is the back-scatter coefficient of the pure water, chlorophyll, SS and DOC respectively. Due to the weak back-scatter of the DOC, we consider the back-scatter of the pure water, chlorophyll and SS only. i.e.

$b_{b}=b_{b_{w}}+b_{b_{p h}}+b_{b_{d}}$ 
The Gordon (1981) model expressed as follows:

$$
R\left(0^{-}\right)=f \frac{b_{b}}{a+b_{b}}
$$

$R\left(0^{-}\right)$is the reflectance below the water surface; $f$ is the invariable, ranges from 0.2 to 0.56 .

With the theory of water spectrum collection, the upward-irradiance $E_{u}\left(0^{-}\right)$of water has water information. The water spectrum change resulted from the water components can be characterized with the downward-irradiance $R\left(0^{-}\right)$:

$$
R\left(0^{-}\right)=E_{u}\left(0^{-}\right) / E_{d}\left(0^{-}\right)
$$

$E_{d}\left(\lambda, 0^{-}\right)$is the down-welling irradiance of the water surface. For

$$
\begin{gathered}
E_{u}\left(0^{-}\right)=Q L_{u}\left(0^{-}\right) \\
L_{w}=L_{u}\left(0^{-}\right) t / n^{2} \\
E_{d}\left(0^{-}\right)=\left(1-\rho_{a w}\right) E d\left(0^{+}\right)
\end{gathered}
$$

While, $Q$ is the optical field distribution parameter, normally with the value $4.0 ; L_{u}\left(0^{-}\right)$is the up-ward irradiance beneath the surface; $L_{w}$ is the water-leaving radiance; $t$ is the penetration coefficient of the gas-water contact, normally with the value 0.98; $n$ is the refraction coefficient of water, normally with the value 1.34; $\rho_{a w}$ is the irradiance reflectance of the gas-water contact (between the 0.04 and 0.06),here we make the variable with the value $0.05 ; E d\left(0^{+}\right)$is the down-welling irradiance just above the surface $^{[4]}$.

With the equation (5), (6),(7),(8), we obtained the equation as following:

$$
R\left(0^{-}\right)=\frac{Q n^{2}}{t\left(1-\rho_{a w}\right)} \frac{L_{w}}{E_{d}\left(0^{+}\right)}
$$

According to the remote sensing reflectance $R_{r s}$

$$
R_{r s}=L_{w} / E_{d}\left(0^{+}\right)
$$

We obtained the equation as following:

$$
R\left(0^{-}\right)=\frac{Q n^{2}}{t\left(1-\rho_{a w}\right)} \frac{L_{w}}{E_{d}\left(0^{+}\right)}=\frac{Q n^{2}}{t\left(1-\rho_{a w}\right)} R_{r s}
$$

Combination the $R_{r s}$ with the total absorption coefficient $a$ and the back-scattering coefficient $b_{b}$, we obtained

$$
R r s=0.049 \frac{b_{b_{w}}+b_{b_{p h}}+b_{b_{d}}}{a_{w}+a_{p h}+a_{d}+a_{g}+b_{b_{w}}+b_{b_{p h}}+b_{b_{d}}}
$$

From the equation, we know that if only the value of the variable $a_{w}$, $a_{p h}{ }^{\prime} a_{d}, a_{g}{ }^{\prime} b_{b_{w}}, b_{b_{p h}}, b_{b_{d}}$ are known ,we can model the remote sensing reflectance of the water with the data in-situ. However, the inherent optical parameters do not have the stationary values. We need to determine the contribution of every water component to total absorption coefficient and the back-scattering coefficient in order to model the remote sensing reflectance of water.

\subsection{The Contribution of Every Water Component to Total} Absorption Coefficient and Back-Scattering Coefficient

The absorption and scattering of the water component is related to their concentration. Morel and Prieur (1977) characterized the absorption with the arithmetic product of the water component concentration and specific absorption coefficient; and characterized the back-scattering with the arithmetic product of volume scattering and backscattering ratio:

$$
\begin{gathered}
a=a_{w}+C_{p h} a_{p h}^{*}+C_{d} a_{d}^{*}+C_{g} a_{g}^{*} \\
b_{b}=\tilde{b}_{b_{w}} b_{w}+\tilde{b}_{b_{p h}} b_{p h}+\tilde{b}_{b_{d}} b_{d}
\end{gathered}
$$

$b_{w}$ is the volume scattering of the pure water; $\tilde{b}_{b_{w}}$ is the backscattering ratio of water; $b_{p h}$ is the volume scattering of the phytoplankton(chlorophyll); $\tilde{b}_{b_{p h}}$ is the backscattering ratio of chlorophyll; $b_{d}$ is the volume scattering of the SS; $\tilde{b}_{b_{d}}$ is the backscattering ratio of SS.

$a_{w}$ is the absorption coefficient of the pure water; $C_{p h}$ is the chlorophyll concentration; $a_{p h}^{*}$ is the specific absorption coefficient of chlorophyll; $C_{d}$ is the SS concentration; $a_{d}^{*}$ is the specific absorption coefficient of SS; $C_{g}$ is the DOC concentration; $a_{g}^{*}$ is the specific absorption coefficient of DOC.

These parameters related to the semi-analytical model can be obtained by experiment in-situ in theory. We choose the reference value from other scholars' research in this study due to the condition we have. 
1.2.1 The Absorption of Every Water Component: Based on the inherent optical properties of every water component, we have researched the absorption coefficient characteristic of phytoplankton (chlorophyll a), non- phytoplankton particulate matter, DOC, and established the absorption coefficient model between the phytoplankton absorption and chlorophyll a concentration, the absorption coefficient model of non-phytoplankton particulate matter, and the absorption coefficient model of DOC. We generalized as following.

- The absorption of the pure water

Many scholars have measured the water absorption. Due to the difference of the water samples, the absorption coefficient of water is different. Morel (1997) gives the absorption coefficient between 200-700nm of fresh water; Pope (1997) gives the absorption coefficient between 380-700nm of pure water; Smith (1981) gives the absorption coefficient between 200-800nm of cleaned natural water. Here, we chose the absorption coefficient between $200-800 \mathrm{~nm}$ of cleaned natural water.

- The absorption of the chlorophyll

With different relative proportion of chl-a,chl-b,chl-c, different phytoplankton component and different condition of light, the absorption coefficient of the phytoplankton is different. However, the absorption of chlorophyll and its concentration has a better non-linear relationship.

For Three Gorges Dam water, polynomial can express the relationship between the chlorophyll a concentration and its absorption coefficient (Gai Li-ya, 2008).Here, we took the typical bands of $443 \mathrm{~nm}$ and $675 \mathrm{~nm}$ for example, the statistic relationship as follows:

$$
\begin{gathered}
a_{p h}(443)=-0.1123\left(C_{p h}\right)^{2}+0.2381 C_{p h}-0.0427 \\
\left(R^{2}=0.7232\right) \\
a_{p h}(675)=-0.0241\left(C_{p h}\right)^{2}+0.0441 C_{p h}+0.0026 \\
\left(R^{2}=0.8247\right)
\end{gathered}
$$

$a_{p h}(443), a_{p h}(675)$ is the absorption coefficient of phytoplankton at band $443,675 \mathrm{~nm}$ respectively, chla is the chlorophyll concentration.

Nevertheless, the statistic relationship between the chlorophyll a and its absorption coefficient at bands $443 \mathrm{~nm}$ and $675 \mathrm{~nm}$ is not obvious due to the less samples number, the mistake in-situ experiment and other factors. Considering the better quality of Three Gorges Dam water (Gai Li-ya, 2008), we chose two different relationships to join the water quality remote sensing monitoring inversion model to inverse the chlorophyll a concentration. One is the polynomial relationship we established based on the water quality of the Three Gorges Dam; the other one is the power exponent model which applies in ocean remote sensing monitoring widely, i.e.

$$
a_{p h}(\lambda)=A[\text { chla }]^{B}
$$

$\lambda$ is the wave length; $a_{p h}(\lambda)$ is the absorption coefficient of phytoplankton; chla is the chlorophyll a concentration; $A, B$ are the fitting parameters.

There are adequate reasons for us to choose the latter relationship. With the measurement data, we know that the chlorophyll a concentration of the Three Gorges Dam water ranges from 0.29 to $1.52 \mathrm{ug} / \mathrm{L}$, and the water quality has been better since the dam builds. The application range of the power exponent model applied in ocean is that the chlorophyll concentration is between $0.02-25 \mathrm{ug} / \mathrm{L}$ (Bricaud,1998). The water quality of the Three Gorges Dam is agree with the applied condition of the power exponent model.

The absorption of the SS

The suspended substance includes organic offal, suspend silts and so on. Compared with the weak information of water, suspended substance has a strong absorption and scattering characteristic (Li Yun-mei, 2006). The constituents of suspended substance minerals in different river and offshore area are different. There is also a difference between the water spectrums. We obtained the absorption coefficient of the suspended substance in Three Gorges Dam by experiment in-situ and established the absorption coefficient model of the suspended substance (Gai Li-ya, 2008).

$$
a_{d}(\lambda)=a_{d}\left(\lambda_{0}\right) \exp \left(-S\left(\lambda-\lambda_{0}\right)\right)
$$

$a_{d}(\lambda)$ is the absorption coefficient of the non-phytoplankton partial matter an the wave $\lambda ; a_{d}\left(\lambda_{0}\right)$ is the absorption coefficient of the non-phytoplankton an the wave $\lambda_{0}$, the value of $\lambda_{0}$ is 440 normally; $s$ is slope, the values of $s$ in different samples is different, and ranges from 0.01010 0.0128,average value is 0.0115 , and the standard deviation is 0.00155 .

Taking \#2, \#19 for example, the non-phytoplankton partial matter as follows:

$$
\begin{aligned}
& a_{d}(\lambda)=a_{d}(440) \exp (-0.01147(\lambda-440)) \\
& a_{d}(\lambda)=a_{d}(440) \exp (-0.01155(\lambda-440))
\end{aligned}
$$

The absorption of the DOC

DOC, which has the unique absorption characteristic, plays an important role to the total absorption spectrum of water. Bricaud (1990) expressed the DOC absorption characteristic with the following equation: 


$$
a_{g}(\lambda)=a_{g}\left(\lambda_{0}\right) \exp \left[S\left(\lambda_{0}-\lambda\right)\right]
$$

$\lambda_{0}$ is the reference wave length; $a_{g}\left(\lambda_{0}\right)$ is the absorption coefficient of DOC at the reference wave length; $S$ is the slop of the DOC absorption coefficient curve. Equation (5.25) is the normally function for appreciating the absorption coefficient of DOC. Many scholars choose different reference wave lengths to fit and calculate the slope of the DOC absorption coefficient curve. Here, we chose the wave length of $440 \mathrm{~nm}$ as the reference wave, with the absorption coefficient of DOC obtained from the experiment in-situ, and fitted the curve in 300-500nm, 501-700nm and 300-700nm bands using least-squares techniques to obtain the value of $S$. Taking \#2 for example, the fitting equation in three different bands as follows:

$$
\text { 300-500n } \quad \begin{aligned}
a_{g}(\lambda) & =0.4235875 \exp [0.01564(440-\lambda)] \\
\left(R^{2}\right. & =0.9928)
\end{aligned}
$$

$$
\begin{gathered}
300-700 \mathrm{~nm}: \quad a_{g}(\lambda)=0.4235875 \exp [0.01562(440-\lambda)] \\
\left(R^{2}=0.9543\right)
\end{gathered}
$$

501-700nm: $\quad a_{g}(\lambda)=0.000002995 \lambda^{2}-0.004261 \lambda+1.606$

$$
\left(R^{2}=0.9903\right)
$$

The correlation between the wave lengths and absorption coefficient of DOC in bands300-500nm, 501-700nm is better, compared with the fitting precision in band $300-700 \mathrm{~nm}$. Therefore, we chose the relationship of the bands $300-500 \mathrm{~nm}$, $501-700 \mathrm{~nm}$ to join the water quality remote sensing inversion model.

1.2.2 The Back-Scattering of Every Water Component: For case I water in ocean, the scattering of water is caused by non-phytoplankton partial matter mainly, And the value of back-scattering $b_{b}$ is very small, i.e. $b_{b}<<a$ (Morel,1977). But for the offshore and inland water in china, such as Huaihai,Taihu and other water area, the information of the scattering coefficient and back-scattering that can be applied is litter due to the larger partial size of pigment particles , the complex scattering characteristic and the strait collection of the scattering, back-scattering data for different components. In addition, some instruments we need are absence. So we did not measure the back-scattering of water components, but did the water quality remote sensing inversion with the reference value that other scholars obtained.

- The scattering of the natural water Smith (1981) gives the back-scattering calculate method of pure water between the bands 200-800nm in 1981 based on the back-scattering of pure water in-situ. Ma Rong-hua (2006), Tang Jun-wu (1997) used this relationship with the algorithm analysis of water components inversion of inland water and water spectrum, and obtained a better inversion result. Here, we also chose this relationship, the relationship as follows (Smith, 1981):

$$
\begin{gathered}
b_{w}(500)=0.00288 \mathrm{~m}^{-1} \\
\frac{b_{w}\left(\lambda_{1}\right)}{b_{w}\left(\lambda_{2}\right)}=\left(\frac{\lambda_{1}}{\lambda_{2}}\right)^{-4.3} \\
\tilde{b}_{b_{w}}=0.5
\end{gathered}
$$

The scattering of the chlorophyll

Based on the hypothesis that the scattering of phytoplankton and absorption has an inversion ratio relationship, the scattering of chlorophyll can be calculated with the equation (30), (31), (32) (Sathyendranath, 1989).

$$
\begin{gathered}
b_{p h}(550)=0.12 C_{p h}^{0.63} \\
b_{p h}(\lambda)=b_{p h}(550) * \frac{a_{p h}(550)}{a_{p h}(\lambda)} \\
\tilde{b}_{b_{p h}}=0.005
\end{gathered}
$$

The scattering of the SS

Many scholars give the calculation methods of suspended substance. Ma Rong-hua (2006) divided the suspended substance into suspended particulate organic matter (SPOM) and suspended particulate inorganic matter (SPIM), and considered that the scattering of the suspended substance is caused by SPIM mainly. He generalized the scattering coefficient calculation equation of suspended substance:

$$
\begin{aligned}
& b_{b_{d}}(\lambda)=b_{b_{S P I M}}(\lambda)=b_{b_{S P I M}}(442)\left(\frac{\lambda}{442}\right)^{B_{B_{b}}} \\
& b_{b_{\text {SPIM }}}(442)=C_{\text {SPIM }} b_{b_{\text {SPIM }}}^{*}(442)+e_{b_{\text {SPIM }}}
\end{aligned}
$$

While, $b_{b_{\text {SPIM }}}(\lambda)$ is the back-scattering coefficient of SPIM; $b_{b_{S P I M}}(442)$ and $B_{B_{b}}$ can be obtained by regression; $C_{\text {SPIM }}$ is the concentration of SPIM; $b^{*}{ }^{*}(442)$ is the specific absorption coefficient of SPIM at $442 \mathrm{~nm} ; e_{b_{b}}$ is the regression residual error. 
Considering the suspended substance was no longer divided in the experiment, so we did not employ this calculation method in our study.

Tang Jun-wu (1997) generalized a calculation method to determine the suspended substance scattering coefficient, which involved the scattering coefficient of suspended substance at $550 \mathrm{~nm}$. We did not employ this calculation method in our study either due to the lack of data.

Peter(2004) generalized a calculation method, which has a better result to calculate the scattering coefficient of suspended substance not only in case I water but also in case II water.

$$
\begin{aligned}
& b_{b_{d}}(\lambda)=0.54 * C_{d}\left(\frac{555}{\lambda}\right)^{0.4} \\
& \tilde{b}_{b_{d}}=0.01833
\end{aligned}
$$

\subsection{Algorithm Integration}

Based on the contribution of water components to total absorption coefficient $a$ and back-scattering $b_{b}$, the relationship between the remote sensing reflectance just above the surface $R_{r s}(\lambda)$ and the inherent optical properties of water can be established. For example, the equation at band $675 \mathrm{~nm}$ as follows.

$$
R r s=0.049 \frac{b_{b}}{a+b_{b}}
$$

While,

$$
\begin{aligned}
b_{b}=b_{b_{w}} & +b_{b_{p h}}+b_{b_{d}} \\
= & 0.5 * b_{w}(500) *\left(\frac{\lambda}{500}\right)^{-4.3}+0.005 * 0.12 C_{p h} 0.63 * \frac{a_{p h}(550)}{a_{p h}(\lambda)} \\
& +0.01833 * 0.54 * C_{d}\left(\frac{555}{\lambda}\right)^{0.4} \\
a= & a_{w}+a_{p h}+a_{d}+a_{g} \\
= & a_{w}-0.0241\left(C_{p h}\right)^{2}+0.0441 C_{p h}+0.0026 \\
& +a_{d}(440) \exp (-0.01147(\lambda-440)) \\
& +0.4235875 \exp [0.01564(440-\lambda)]
\end{aligned}
$$

With the remote sensing reflectance just above the surface $R_{r s}(\lambda)$ in experiment in-situ, we established the non-linear system of equations using the variable $R_{r s}(\lambda)$ of two random bands at one sample. The equation is as followed.

$$
\begin{aligned}
& \operatorname{Rrs}\left(\lambda_{1}\right)=0.049 \frac{b_{b}\left(\lambda_{1}\right)}{a\left(\lambda_{1}\right)+b_{b}\left(\lambda_{1}\right)} \\
& \operatorname{Rrs}\left(\lambda_{2}\right)=0.049 \frac{b_{b}\left(\lambda_{2}\right)}{a\left(\lambda_{2}\right)+b_{b}\left(\lambda_{2}\right)}
\end{aligned}
$$

There are only two indeterminate variables in this equation, so we can calculate the concentration of chlorophyll a and suspended substance.

\section{ALGORITHM APPLICATIONS}

\subsection{The Precision Analysis of Model}

Theoretically speaking, the applicability of the analytical model is widely than that of the experiential model. However, we quoted the reference values of some variables, considering the correlation between the chlorophyll a concentration and the absorption is weak, so we chose the bands that the correlation between the chlorophyll a concentration and the absorption is better to inverse the water components concentration. With the analyses of the correlation between the water components concentration and band reflection, the correlation between the chlorophyll a and band675nm, 443nm is higher. The precision of the model is analyzed with the measured data on 15 samples in-situ.

Here, we defined the inversion model that used the polynomial equation expressing the chla concentration and the absorption as model $(-)$,and defined the inversion model that used the power exponent equation expressing the chla concentration and the absorption as $\operatorname{model}(\stackrel{\rightarrow}{\rightarrow}$.

\section{Model $(\rightarrow)$}

The inversion error of chla on odd number samples is shown in Table 1.The statistical chart of the chla concentration inversion error is shown in Fig 1.

\begin{tabular}{|c|c|c|c|}
\hline Samples & $\begin{array}{c}\text { Chla Concentration } \\
\text { Measured } \\
\text { (ug/l) }\end{array}$ & $\begin{array}{c}\text { chla Concentration } \\
\text { Calculated } \\
\text { (ug/l) }\end{array}$ & $\begin{array}{c}\text { Error } \\
(\%)\end{array}$ \\
\hline S1 & 2.98 & 1.66 & -44.14 \\
\hline S3 & 0.47 & 1.12 & -139.96 \\
\hline S5 & 0.53 & 1.17 & -121.62 \\
\hline S7 & 0.32 & 0.45 & 41.5625 \\
\hline S9 & 0.64 & 0.35 & -44.44 \\
\hline S13 & 0.64 & 0.18 & -70.42 \\
\hline S15 & 1.19 & 0.36 & -68.98 \\
\hline S17 & 1.15 & 0.29 & -74.11 \\
\hline S19 & 0.56 & 0.73 & 31.50 \\
\hline S21 & 1.52 & 0.47 & 68.68 \\
\hline S23 & 0.31 & 0.27 & -12.26 \\
\hline S25 & 0.61 & 0.44 & -28.05 \\
\hline S27 & 0.68 & 1.13 & 65.75 \\
\hline S29 & 0.29 & 0.36 & 23.79 \\
\hline
\end{tabular}

Table 1 The Retrieval Error of the Chla Concentration at odd samples 


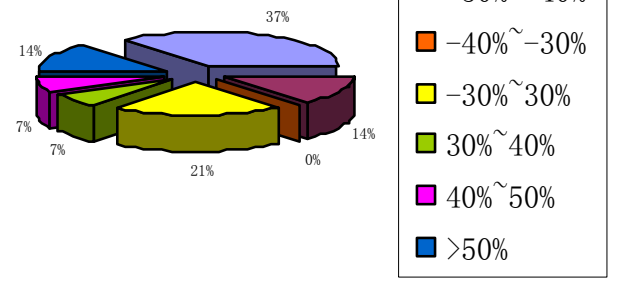

Figure 1 The Retrieval Error Distribution Map of the Chla Concentration at odd samples

With the statistical data, it is not feasible for using the model $(-)$ to inverse the chla concentration in Three Gorges Dam. The maximum inversion error is $-139.96 \%$, and the minimum inversion error is $-12.26 \%$, and the mean error is $61.05 \%$, and the relative error of the majority points is concentrated between $\pm 80 \%$ and $\pm 40 \%$. Analysizing the reason, the first one is possible due to the lack of the chla concentration samples in-situ, which resulted in the weak correlation between the chla concentration and the absorption coefficients; the other one is possible due to the parameter in the model, such as the $f, Q$,which is influenced a lot by the environmental condition.

\section{Model $(\rightarrow)$}

The inversion error of chla on odd number samples is shown in Table 2.The statistical chart of the chla concentration inversion error is shown in Fig 2.

\begin{tabular}{|c|c|c|c|}
\hline Samples & $\begin{array}{c}\text { Chla } \\
\text { Concentration } \\
\text { Measured } \\
\text { (ug/l) }\end{array}$ & $\begin{array}{c}\text { chla } \\
\text { Concentration } \\
\text { Calculated } \\
\text { (ug/l })\end{array}$ & $\begin{array}{c}\text { Error } \\
(\%)\end{array}$ \\
\hline S3 & 0.47 & 1.76 & 275.04 \\
\hline S5 & 0.53 & 0.85 & 61.64 \\
\hline S7 & 0.32 & 0.93 & 192.43 \\
\hline S9 & 0.64 & 0.15 & -75.19 \\
\hline S13 & 0.64 & 0.85 & 33.14 \\
\hline S15 & 1.19 & 0.22 & -80.93 \\
\hline S17 & 1.15 & 0.15 & -86.50 \\
\hline S19 & 0.56 & 1.95 & 249.37 \\
\hline S21 & 1.52 & 1.98 & 30.59 \\
\hline S23 & 0.31 & 0.96 & 211.16 \\
\hline S25 & 0.61 & 0.93 & 53.62 \\
\hline S27 & 0.68 & 1.24 & 83.00 \\
\hline S29 & 0.29 & 0.84 & 205.03 \\
\hline
\end{tabular}

Table 2 The Retrieval Error of the Chla Concentration at odd samples

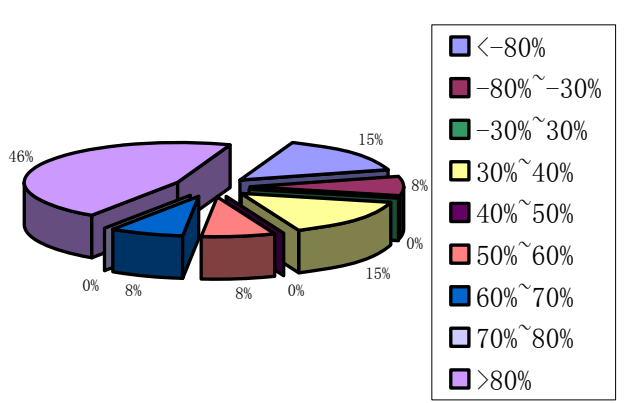

Figure 2 The Retrieval Error Distribution Map of the Chla Concentration at odd samples

With the statistical data, the chla concentration inversion maximum error is $275.04 \%$, and the minimum error is $30.59 \%$,the mean error is $89.15 \%$.There are about $46 \%$ of the samples, which the inversion error is up to $80 \%$.i.e. it does not improve the inversion precision to use the $\operatorname{model}(\rightarrow)$ which is joined with the power exponent to express the relationship of the chla concentration and the absorption coefficient .In other way, though the chla concentration in Three Gorges Dam is not high ,it is different from the water in ocean. We can not replace the absorption characteristic in Three Gorges Dam with that in ocean.

Considering the precision of the two models ,we choose the model $(\rightarrow)$ to inverse the chla concentration in Three Gorges Dam.

\subsection{Algorithm Applications}

Despite CHRIS is a hyperspectral sensor, of which the spectrum resolution is above $10 \mathrm{~nm}$, the band design of it is rougher, compared with the spectrum width of Handhold Field ASD spectrometer (spectrum resolution is above $1 \mathrm{~nm}$ ). So there is a coupling between the bands chosen of CHRIS and the retrieval algorithm based on the data Handhold Field ASD measured, i.e. the band design of CHRIS is not satisfied the retrieval algorithm accurately. As a matter of fact, we chose band 2(the mid band is $442 \mathrm{~nm}$ ) corresponding to $443 \mathrm{~nm}$, band 11 (the mid band is $672 \mathrm{~nm}$ ) corresponding to $675 \mathrm{~nm}$, and apply the retrieval model $\rightarrow$ on the CHRIS image which we obtained on April, $17^{\text {th }}$, 2007.The chla concentration in Three Gorges Dam was retrieval led and was shown in table 3. 


\begin{tabular}{|c|c|c|c|}
\hline Samples & $\begin{array}{c}\text { Chla } \\
\text { Concentration } \\
\text { Measured } \\
\text { (ug/l) }\end{array}$ & $\begin{array}{c}\text { chla } \\
\text { Concentration } \\
\text { Calculated } \\
\text { (ug/l) }\end{array}$ & $\begin{array}{c}\text { Error } \\
(\%)\end{array}$ \\
\hline S3 & 0.47 & 0.96 & 104.25 \\
\hline S5 & 0.53 & 1.08 & 103.78 \\
\hline S7 & 0.32 & 0.39 & 21.88 \\
\hline S9 & 0.64 & 0.47 & -26.56 \\
\hline S13 & 0.64 & 0.23 & -64.06 \\
\hline S15 & 1.19 & 0.45 & -62.18 \\
\hline S17 & 1.15 & 0.31 & -73.04 \\
\hline S19 & 0.56 & 0.74 & 32.14 \\
\hline S21 & 1.52 & 0.46 & 69.73 \\
\hline S23 & 0.31 & 0.28 & 9.68 \\
\hline S25 & 0.61 & 0.52 & -14.75 \\
\hline S27 & 0.68 & 1.04 & 52.94 \\
\hline S29 & 0.29 & 0.34 & 17.24 \\
\hline
\end{tabular}

Table 3 The Retrieval Error of the Chla Concentration at odd samples

As we can see, the result is not ideal. Most of the inversion error is beyond $60 \%$.Some of them is up to $100 \%$.It is pointed that it is better to apply the synchronous data measured in-situ. While in fact, we applied the measured data on November $7^{\text {th }}-8^{\text {th }}, 2007$ to compare with the CHRIS image on April, 17th, 2007. In November, it is cold and the mobility of the phytoplankton becomes weak, and the velocity of water flow is slow which lead the suspended matter deposit in the bottom of the river. So it is not surprising that the band reflectance we measured is low. While in April, a warmer spring, varies of phytoplankton begin to grow and the mobility of them is strong, most of the suspended matter in water is in suspended state which is influenced with the mold rain. The differences of the season result that the spectrum characteristic and the absorption, scattering characteristic of water components have changed a lot. Therefore, the application of the model need to certificate further.

\section{CONCLUSIONS}

Based on the research we have done, we analyzed the contribution of water components to the total absorption, scattering, established the water quality remote sensing model in Three Gorges Dam, calculated the Chla concentration in Three Gorges Dam using the algebra method. The results show that, it is not advisable for using the semi-analytical to inverse the chla concentration. We should explore the new methods or models to improve the inversion precision, especially to establish the best basal model to express the relationship of the chla concentration and the absorption coefficient.

For our inland water, especially the water in Three Gorges Dam, which is not only different from the ocean, but also different from the lake, river in inland, it is need to accumulate abundant, reliable data in-situ to develop the semi-analytical algorithm to apply. It is not only that the indeterminacy factor influencing the measured precision in-situ is so much that it is difficult to obtain the high-grade data, but also the terrigenous importation which make the work more difficult. Therefore, we should to enhance the accumulation of the data in-situ, and standardize the measured operation in-situ, perfect the measured methods that we have known, reduce the error that created in the measured processing due to the anthropogenic factor, so as to increase the accuracy of the data obtained in-situ.

\section{REFERENCE}

Bricaud A, Morel A, Babin M, et al. Variations of light absorption by suspended particles with chlorophyll a concentration in oceanic (case I) waters : analysis and implication for bio-optical models [J], Journal of Geophysical Research, 1998, 103: 31033-31044.

Bricaud A and Stranmski D. Spectral absorption coefficients of living phytoplankton and nonalgal biogenous matter: A comparison between the Preu upwelling area and the Sargasso Sea [J], Limnology and Oceanography, 1990, 35(3): 562-582.

Gailiya.The study of the inversion algorithms for inland water components---In Three Gorges Dam water [D], Shan Dong University of Science and Technology, 2008.

Gordon H R, Brown O B, Jacobs M. Computed relationships between the inherent and apparent optical properties of a flat homogeneous ocean [J], Applied Optics, 1981, 14(2): 417-427. Li Tong-ji, Zhu Jian-hua, Cheng Qing-lian. Semi-Empirical Analysis Bio-Algorithm Research of Yellow-East Sea in spring [J], Ocean Technology, 2006, 25(1):83-88.

Li Yun-mei, Huang Jia-zhu, Wei Yu-chun, et al. Inversing Chlorophyll Concentration of TaiHu Lake by Analytic Model[J], Journal of Remote Sensing, 2006, 10(2): 169-175.

Liu Chan-de, He bao-yin. Processing in Water Monitoring Using Remote Sensing [J], Science Advance and Learned Review, 2005, 27(5), 40-45.

Ma Rong-hua,Tang Jun-wu.Remote sensing parameters acquisition and algorithm analysis of lake color[J],Advances in Water Sciences,2006,17(5):720-726.

Morel A, Prieur L. Analysis of variation in ocean color [J], Limnology and Ocean logy, 1977, 22: 709-722.

Peter K, Steve D B, Youngje P. Retrieval of oceanic constituents from ocean color using simulated annealing [J], Proc. Of the 2nd CHRIS/PROBA workshop, ESA/ESRIN, Frascati, Italy, 28-30 April 2004.

Pope R M, Fry E S. Absorption spectrum (380-700nm) of pure water II Integrating cavity measurements [J], Applied Optics, 1997, 36(33): 8710-8723. 
Ren Jing-ping, Zhao Jin-ping. Processing and Prospect of Ocean Color Remote Sensing in Case2 Waters [J], Advance in Earth Sciences, 2002, 17(3):363-371.

Sathyendranath S, Prieur L, Morel A. A three-component model of ocean color and its application to remote sensing of phytoplankton pigments in coastal waters[J ], International Journal of Remote Sensing, 1989, 10: 1373-1394.

Smith R C, Baker K S. Optical properties of the clearest natural waters(200-800nm)[J], Applied Optics, 1981, 20(2): 177-184. Tang Jun-wu,Tian Guo-liang. Ocean Color Analysis and an Algorithm for the Retrieval of Multiconstituents Based on
Remote Sensing Reflectance[J],Journal of Remote Sensing,1997,1(4),252-256.

\section{FOUNDATION}

Funded by China's National Social Development Research Program funded by the Ministry of Science and Technology (approved No. 2004DIB3J107), Key Laboratory of Geo-informatics of State Bureau of Surveying and Mapping (A1714), and State key Laboratory of Remote Sensing Science (LRSS). 